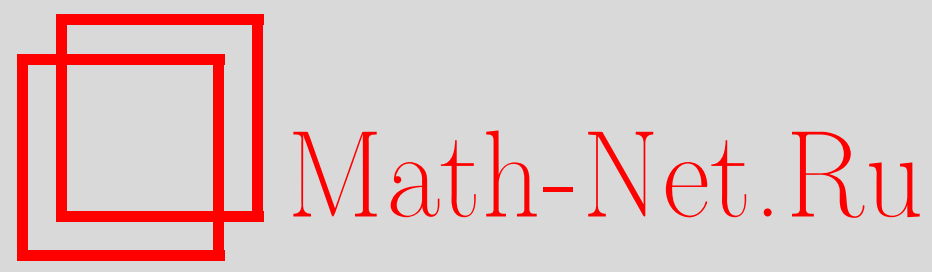

А. Ю. Колесов, Н. Х. Розов, Существование решений с точками поворота у нелинейных сингулярно возмущенных краевых задач, Матем. заметки, 2000, том 67, выпуск 4, 520524

DOI: https://doi.org/10.4213/mzm867

Использование Общероссийского математического портала Math-Net.Ru подразумевает, что вы прочитали и согласны с пользовательским соглашением http://www.mathnet.ru/rus/agreement

Параметры загрузки:

IP : 54.237 .206 .68

26 апреля 2023 г., $17: 16: 47$

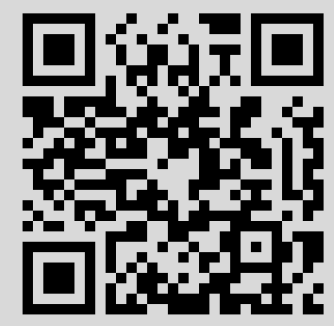




\section{СУЩЕСТВОВАНИЕ РЕШЕНИЙ С ТОЧКАМИ ПОВОРОТА У НЕЛИНЕЙНЫХ СИНГУЛЯРНО ВОЗМУЩЕННЫХ \\ КРАЕВЫХ ЗАДАЧ}

\section{А. Ю. Колесов, Н. Х. Розов}

На отрезке $0 \leqslant x \leqslant 1$ рассматривается сингулярно возмущенная краевая задача

$$
\begin{gathered}
\varepsilon u^{\prime \prime}=f\left(x, u, u^{\prime}\right), \quad 0<\varepsilon \ll 1, \\
g_{j}\left(\left.u\right|_{x=0},\left.u\right|_{x=1},\left.u^{\prime}\right|_{x=0},\left.u^{\prime}\right|_{x=1}\right)=0, \quad j=1,2 .
\end{gathered}
$$

Изучается проблема существования и единственности ее решения $u(x, \varepsilon)$ со следуюшими свойствами: $u(x, \varepsilon) \rightarrow u_{0}(x)$ при $\varepsilon \rightarrow 0$ равномерно по $x \in[0,1]$, где $u_{0}(x) \in$ $C^{\infty}[0,1]$ - решение вырожденного уравнения $f\left(x, u, u^{\prime}\right)=0$; существует такая точка $x_{0} \in(0,1)$, что $a\left(x_{0}\right)=0, a^{\prime}\left(x_{0}\right)>0, a(x)<0$ при $0 \leqslant x<x_{0}, a(x)>0$ при $x_{0}<x \leqslant 1$, где $a(x)=f_{v}^{\prime}\left(x, u_{0}(x), u_{0}^{\prime}(x)\right)$.

Библиография: 3 названия.

На отрезке $0 \leqslant x \leqslant 1$ рассмотрим сингулярно возмущенную краевую задачу

$$
\begin{gathered}
\varepsilon u^{\prime}=f\left(x, u, u^{\prime}\right), \quad 0<\varepsilon \ll 1, \quad '=\frac{d}{d x}, \\
g_{j}\left(\left.u\right|_{x=0},\left.u\right|_{x=1},\left.u^{\prime}\right|_{x=0},\left.u^{\prime}\right|_{x=1}\right)=0, \quad j=1,2,
\end{gathered}
$$

где функции $f(x, u, v), g_{j}\left(u_{1}, u_{2}, v_{1}, v_{2}\right), j=1,2$, бесконечно дифференцируемы по совокупности переменных $(x, u, v) \in[0,1] \times \mathbb{R} \times \mathbb{R},\left(u_{1}, u_{2}, v_{1}, v_{2}\right) \in \mathbb{R}^{4}$. Будем интересоваться существованием решения $u(x, \varepsilon)$, для которого равномерно по $x \in[0,1]$ справедливо предельное равенство

$$
\lim _{\varepsilon \rightarrow 0} u(x, \varepsilon)=u_{0}(x)
$$

здесь $u_{0}(x) \in C^{\infty}[0,1]$ - решение вырожденного уравнения

$$
f\left(x, u, u^{\prime}\right)=0
$$

имеющее точку поворота. Последнее означает наличие такой точки $x_{0} \in[0,1]$, что

$$
\begin{aligned}
a\left(x_{0}\right) & =0, \quad a^{\prime}\left(x_{0}\right)>0, \\
a(x) & <0 \quad \text { при } 0 \leqslant x<x_{0}, \quad a(x)>0 \quad \text { при } \quad x_{0}<x \leqslant 1,
\end{aligned}
$$

Работа выполнена при поддержке Российского фонда фундаментальных исследований и фонда INTAS. 
где $a(x)=f_{v}^{\prime}\left(x, u_{0}(x), u_{0}^{\prime}(x)\right)$.

Отметим, что сушествование нужного решения $u_{0}(x)$ у уравнения $(3)$ не очевидно и требует дополнительных условий на функцию $f$. Кроме того, необходимо исследовать и проблему существования решения $u(x, \varepsilon)$ исходной задачи $(1)$ с асимптотическим поведением (2). Однако, как показано ниже, обе эти проблемы сводятся к вопросу о существовании траекторий-уток у некоторой вспомогательной системы дифференциальных уравнений.

Сделаем следующие дополнительные предположения.

УСловиЕ 1. При каждом $x \in[0,1]$ система уравнений

$$
f(x, u, v)=0, \quad f_{v}^{\prime}(x, u, v)=0
$$

имеет единственное решение $(u, v)=(z(x), w(x)) \in C^{\infty}[0,1]$, причем

$$
f_{u}^{\prime}(x, z(x), w(x)) \neq 0, \quad f_{v v}^{\prime \prime}(x, z(x), w(x)) \neq 0
$$

УСЛовИЕ 2. Существует такое $x_{0} \in(0,1)$, что $\varphi\left(x_{0}\right)=0, \varphi^{\prime}\left(x_{0}\right) \neq 0$, әде $\varphi(x)=w(x)+f_{x}^{\prime}(x, z(x), w(x)) / f_{u}^{\prime}(x, z(x), w(x))$.

Введем в рассмотрение квадратичньй полином $P(\lambda)=\lambda^{2}+\alpha \lambda+\beta, \alpha=f_{u}^{\prime}\left(x_{0}, z\left(x_{0}\right)\right.$, $\left.w\left(x_{0}\right)\right), \beta=f_{u}^{\prime}\left(x_{0}, z\left(x_{0}\right), w\left(x_{0}\right)\right) f_{v v}^{\prime \prime}\left(x_{0}, z\left(x_{0}\right), w\left(x_{0}\right)\right) \varphi^{\prime}\left(x_{0}\right)$.

УСЛовИЕ 3. Полином $P(\lambda)$ имеет два вещественных корня $\lambda_{1}<\lambda_{2}$, причем $\lambda_{2}>0$.

Покажем, что сформулированные условия позволяют определить требуемое решение $u_{0}(x)$ уравнения (3) локально (в некоторой достаточно малой окрестности фигурирующей в условии 2 точки $x_{0}$ ). С этой целью сначала, опираясь на первое неравенство (6) и теорему о неявной функции, из первого уравнения (5) находим функцию

$$
\begin{aligned}
u & =\Psi(x, v): \Psi\left(x_{0}, w\left(x_{0}\right)\right)=z\left(x_{0}\right) \\
\Psi_{x}^{\prime}\left(x_{0}, w\left(x_{0}\right)\right) & =-\frac{f_{x}^{\prime}\left(x_{0}, z\left(x_{0}\right), w\left(x_{0}\right)\right)}{f_{u}^{\prime}\left(x_{0}, z\left(x_{0}\right), w\left(x_{0}\right)\right)}, \quad \Psi_{v}^{\prime}(x, w(x)) \equiv 0 .
\end{aligned}
$$

Затем, дифференцируя равенство $u=\Psi(x, v)$ по $x$ и полагая $u^{\prime}=v$, перейдем от $(3) \mathrm{k}$ эквивалентному уравнению $v^{\prime}=\left(v-\Psi_{x}^{\prime}(x, v)\right) / \Psi_{v}^{\prime}(x, v)$.

Следующий этап связан с переходом от этого уравнения к эквивалентной ему системе на плоскости $\dot{x}=\Psi_{v}^{\prime}(x, v), \dot{v}=v-\Psi_{x}^{\prime}(x, v),^{\cdot}=d / d t$, от которой, в свою очередь, посредством замены $v=w(x)+\xi$ перейдем к системе

$$
\begin{aligned}
& \dot{x}=\Psi_{v}^{\prime}(x, w(x)+\xi) \\
& \dot{\xi}=w(x)+\xi-\Psi_{x}^{\prime}(x, w(x)+\xi)-w^{\prime}(x) \Psi_{v}^{\prime}(x, w(x)+\xi) .
\end{aligned}
$$

Из указанных вьше условий и из свойств функции $\Psi$ (см. (7)) заключаем, что система (8) имеет состояние равновесия $x=x_{0}, \xi=0$ с характеристическими показателями (корнями полинома) $\mu_{j}=-\lambda_{j} /\left(f_{u}^{\prime}\left(x_{0}, z\left(x_{0}\right), w\left(x_{0}\right)\right)\right), j=1,2$. А так как (условия 2 и 3) $\lambda_{1} \lambda_{2}=\beta \neq 0$, то это положение равновесия является либо седлом (при $\left.\lambda_{1}<0\right)$, 
либо узлом (при $\left.\lambda_{1}>0\right)$. Но в любом случае интегральное многообразие системы (8), отвечающее наибольшему по модулю показателю $\mu_{2}$, задается равенством

$$
\xi=\xi_{0}(x): \xi_{0}\left(x_{0}\right)=0, \quad \xi_{0}^{\prime}\left(x_{0}\right)=\frac{\lambda_{2}}{f_{v v}^{\prime \prime}\left(x_{0}, z\left(x_{0}\right), w\left(x_{0}\right)\right)}
$$

где функция $\xi_{0}(x) \in C^{\infty}$ определена в некоторой достаточно малой окрестности точки $x=x_{0}$ (см., например, [1]).

Итак, при условиях 1-3 уравнение (3) в некоторой малой окрестности точки $x=x_{0}$ имеет решение

$$
u_{0}(x)=z\left(x_{0}\right)+\int_{x_{0}}^{x}\left[w(s)+\xi_{0}(s)\right] d s
$$

со свойствами (4), причем для функции $a(x)$ справедливы равенства $a\left(x_{0}\right)=0, a^{\prime}\left(x_{0}\right)=$ $\lambda_{2}>0$. Однако, вообще говоря, такое решение не обязано существовать на всем отрезке $0 \leqslant x \leqslant 1$ (ибо соответствующее интегральное многообразие системы (8) равенством $(9)$ при всех $0 \leqslant x \leqslant 1$ задается не всегда). Поэтому возникает необходимость в еще одном предположении.

УСЛовИЕ 4. Решение (10) уравнения (3) продолэсается на весь отрезок $0 \leqslant x \leqslant 1$ с сохранением свойств (4).

Условия 1-4 обеспечивают существование требуемого решения $u_{0}(x)$ вырожденного уравнения (3). Следуюшая групп предложений нужна для того, чтобы гарантировать существование решения $u(x, \varepsilon)$ исходной краевой задачи $(1)$ с данным нулевьм приближением $u_{0}(x)$. Положим

$$
\begin{aligned}
& G_{j}\left(v_{1}, v_{2}\right)=g_{j}\left(u_{0}(0), u_{0}(1), v_{1}, v_{2}\right), \quad j=1,2 \\
& F_{1}(v)=f\left(0, u_{0}(0), v\right), \quad F_{2}(v)=f\left(1, u_{0}(1), v\right) .
\end{aligned}
$$

УСловие 5. Система

$$
G_{j}\left(v_{1}, v_{2}\right)=0, \quad j=1,2,
$$

имеет единственное решение $\left(v_{1}, v_{2}\right)=\left(v_{1}^{0}, v_{2}^{0}\right)$, причем отличен от нуля соответствующий якобиан.

УСловиЕ 6. Решение $v_{j}(t), j=1,2$, задач Коши

$$
\dot{v}=F_{j}(v),\left.\quad v\right|_{t=0}=v_{j}^{0}, \quad j=1,2,
$$

определены соответственно на полуосях $t \geqslant 0, t \leqslant 0, u$ выполнены равенства

$$
\lim _{t \rightarrow+\infty} v_{1}(t)=u_{0}^{\prime}(0), \quad \lim _{t \rightarrow-\infty} v_{2}(t)=u_{0}^{\prime}(1) .
$$

Содержательность условий 1-6 проиллюстрируем на простом примере: $f=v^{2}+u-$ $5(x-1 / 2)^{2}, g_{1}=v_{1}, g_{2}=v_{2}$. Действительно, условия 1-5 здесь очевидньм образом выполняются, причем $u_{0}(x)=(x-1 / 2)^{2}$. Кроме того, в данном случае $v_{1}(t)=v_{2}(t)=v(t)$, где $v(t)$ - решение задачи Коши $\dot{v}=v^{2}-1,\left.v\right|_{t=0}=0$, а значит, вьполняется и условие 6 . 
ТЕорема. При сформулированных условиях и при всех достаточно малых $\varepsilon>0$ решение и $(x, \varepsilon)$ краевой задачи (1), удовлетворяющее предельному неравенству (2), существует и единственно.

ДокАЗАТЕЛЬСтво. Отметим, что в теореме речь идет о единственности решения с фиксированньм нулевым приближением $u_{0}(x)$, в то время как количество самих таких функций $u_{0}(x)$ определяется в первую очередь количеством нулей функции $\varphi(x)$ (см. условие 2), а значит, может быть любым.

Введем в рассмотрение релаксационную систему

$$
\dot{u}=v, \quad \varepsilon \dot{v}=f(x, u, v), \quad \dot{x}=1
$$

и отвечающую ей вырожденную систему

$$
\dot{u}=v, \quad f(x, u, v)=0, \quad \dot{x}=1 .
$$

Фиксировав произвольно малое $\delta_{1}>0$, заметим, что поскольку на кривой $\Gamma_{0}=\left\{\left(u_{0}(x)\right.\right.$, $\left.\left.u_{0}^{\prime}(x), x\right): 0 \leqslant x \leqslant x_{0}-\delta_{1}\right\}$ вьполняется неравенство $f_{v}^{\prime}<0$, то в ее окрестности можно разрешить относительно $v$ второе уравнение $(15): v=\Phi(x, u)$. Таким образом, система (15) в этом случае эквивалентна скалярному уравнению, которое мы дополним начальньм условием:

$$
u^{\prime}=\Phi(x, u),\left.\quad u\right|_{x=0}=u_{*} \in\left[u_{0}(0)-\delta_{2}, u_{0}(0)+\delta_{2}\right], \quad \delta_{2}>0 .
$$

Заметим, что при подходящем уменьшении $\delta_{2}$ каждое решение задачи (16) существует на отрезке $0 \leqslant x \leqslant x_{0}-\delta_{1}$. Действительно, при $\delta_{2}=0$ решением задачи (16) является функция $u_{0}(x)$, определенная (согласно условию 4 ) при всех $0 \leqslant x \leqslant 1$. А отсюда требуемьй факт вытекает очевидным образом.

Введем две интегральные поверхности $S_{0}\left(u_{*}, \varepsilon\right)$ и $S_{1}\left(u_{* *}, \varepsilon\right)$, которые порождаются траекториями системы (14) с начальными условиями

$$
\begin{array}{lll}
\left.u\right|_{t=0}=u_{*} \in\left[u_{0}(0)-\delta_{2}, u_{0}(0)+\delta_{2}\right], & \left.v\right|_{t=0}=v_{*}, & \left.x\right|_{t=0}=0, \\
\left.u\right|_{t=0}=u_{* *} \in\left[u_{0}(1)-\delta_{2}, u_{0}(1)+\delta_{2}\right], & \left.v\right|_{t=0}=v_{* *}, & \left.x\right|_{t=0}=1,
\end{array}
$$

рассматриваемыми при $t \geqslant 0$ и $t \leqslant 0$ соответственно. Здесь $\left(v_{*}, v_{* *}\right)$ - решение системы (12), в которой функции $G_{j}, j=1,2$, определены равенствами (11) при замене $u_{0}(0)$ на $u_{*}$ и $u_{0}(1)$ на $u_{* *}$ (в силу условия 5 и малости $\delta_{2}$ такое решение существует и единственно).

Опишем, например, асимптотическое поведение при увеличении $t$ траекторий системы (14) с начальными условиями (17). Согласно результатам монографии [2] сначала происходит быстрое движение в асимптотически малой окрестности прямой $x=0$, $u=u_{*}$, описьваемой задачей Коши $(\tau=\varepsilon t)$

$$
\frac{d v}{d \tau}=f\left(0, u_{*}, v\right),\left.\quad v\right|_{\tau=0}=v_{*} .
$$

Подчеркнем, что в силу условия 6 , а также близости $u_{*}$ к $u_{0}(1)$ и $v_{*}$ к $v_{1}^{0}$ решение $v(\tau)$ этой задачи близко к решению первой из задач Коши (13), а значит, оно определено при 
всех $\tau \geqslant 0$ и $v(\tau) \rightarrow \Phi\left(0, u_{*}\right)$ при $\tau \rightarrow+\infty$. Таким образом, происходит "падение" каждой из траекторий семейства $S_{0}$ на устойчивое многообразие медленного движения $v=\Phi(x, u)$. Затем движение продолжается в асимптотически малой его окрестности по закону (16) вплоть до плоскости $x=x_{0}-\delta_{1}$.

Итак, поверхность $S_{0}$ пересекает общим образом плоскость $x=x_{0}-\delta_{1}$. Положим $\Sigma_{0}=S_{0} \cap\left\{x=x_{0}-\delta_{1}\right\}$, а через $\Sigma_{1}$ обозначим пересечение поверхности $S_{1}$ с плоскостью $x=x_{0}+\delta_{1}$ (поведение траекторий системы (14) с начальньми условиями $(18)$ при $t \leqslant 0$ аналогично описанному вьше). Наконец, воспользуемся результатами монографии [3], из которых вытекает существование единственной траектории-утки

$$
L(\varepsilon)=\left\{(u(x, \varepsilon), v(x, \varepsilon), x), x_{0}-\delta_{1} \leqslant x \leqslant x_{0}+\delta_{1}\right\}
$$

соединяющей $\Sigma_{0}$ и $\Sigma_{1}$. Ее компонента $u(x, \varepsilon)$ после продолжения на весь отрезок $0 \leqslant$ $x \leqslant 1$, очевидно, и будет искомым решением $u(x, \varepsilon)$ краевой задачи $(1)$.

Остается добавить, что (как показано в [3]) равномерно по $0 \leqslant x \leqslant 1$ справедливо асимптотическое равенство $u(x, \varepsilon)=u_{0}(x)+O(\varepsilon)$. Теорема доказана.

В заключение подчеркнем, что условия 1-6 предполагают нетривиальную зависимость функции $f$ от всех ее аргументов, а по переменной $v$ она должна быть существенно нелинейной. Поэтому из нашего рассмотрения заведомо выпадает важный частньй случай $f \equiv A(x, u) v+B(x, u)$. Однако для этого случая также возможно построение аналогичной теории.

\section{СПИСОК ЦИТИРОВАННОЙ ЛИТЕРАТУРЫ}

[1] Хартман Ф. Обыкновенные дифференциальные уравнения. М.: Мир, 1970.

[2] Васильева А. Б., Бутузов В. Ф. Асимптотические разложения решений сингулярно возмущенных уравнений. М.: Наука, 1973.

[3] Мищенко Е. Ф., Колесов Ю. С., Колесов А. Ю., Розов Н. Х. Периодические движения и бифуркационные процессы в сингулярно возмущенных системах. М.: Наука, 1995. 